\title{
FOREST FIRE DETECTION SYSTEM USING IOT
}

\author{
Mrs. Pavitra N \\ Assistant Professor \\ Department of Computer Science \& Engineering Presidency University \\ Bengaluru, India \\ Sania Khan, Seeksha Jain, Anusha MN, Y Pavan Kalyan \\ Department of Computer Science \& Engineering Presidency University \\ Bengaluru, India
}

\begin{abstract}
Forest Fire Detection System focuses on developing a system that ensures the safety of the forest by sending an SMS alert to the forest officials for rescue as soon as a fire is detected. This is implemented using IoT and Image Processing technologies. The proposed system mainly focuses on building a fire detection system using ESP 32 Microcontroller which is connected to a Temperature sensor, IR sensor, Neo 6m GPS Module, and Sim800L GSM Module. Initially, various sensors will be placed in different parts of the forest to detect the fire and once any fire is detected, the GPS module will be used to trace the location. Using the GSM module, the fire detected location is sent via an SMS in the form of a link. The drone will then be sent to this location to capture and send the images to the forest officials to analyze the intensity of the fire.

The system is made smarter by linking the monitoring process to a webpage in which the forest officials can log in and have a track on the visualization of fire through image processing by using the Sobel Edge detection algorithm (Django Application).
\end{abstract}

Keywords-ESP 32, IR sensor, SMS, Neo 6m GPS Module, Sim800L GSM Module, Sobel Edge Algorithm.

\section{INTRODUCTION}

The forest plays a very important role in any country and mankind as a whole. They contribute to the environmental, economic, and social well- being of the country. The forests are very crucial for the survival of the wildlife and also play an important role in the food chain to maintain the ecological balance. All of us depend on the forest for our survival which includes activities from the air breath to the wood used. Forest not only provides habitat for the animal's livelihood for humans but they also help in the watershed protection, soil erosion prevention and mitigation of climate change.

Forest fire detection system is used to detect forest fire and to send the required amount of help to stop fire after processing of fire images captured by the drone. The drone images are processed on the Django Application using Sobel edge Algorithm.

The entire process is divided into two modules 1.GPS module and 2. Drone Module.

\section{LITERATURE REVIEW}

Ignacio Bosch, Soledad Gomez, Luis Vergara et al. [1] have proposed a paper based on a scheme of infrared sensors. This scheme based on infrared image processing performs the immediate detection of any fire in the forest to determine the presence or absence of fire. Sensor networks are widely used and help the human capabilities to monitor large forest areas. This paper describes a scheme for automatic forest surveillance with the help of IR sensors. The paper describes only about detecting the fire and sending images of detected fire using image processing

Yogesh Deshpande, Krishi Savla, Crispin Lobo, Jahnavi Patel \&amp; prof. Shivani Bhattacharjee et al. [2] has proposed a paper on Forest monitoring systems using sensors, wireless communication, and image processing. The forest monitoring system will keep a track of the conditions that are good for the fauna of the forest and collect data which includes temperature, humidity, entry and exit of animals and the various parts of the forest

Diyana Kinaneva, Georgi Hristov, Jordan Raychev and Plamen Zahariev et al. in [3] has proposed a paper whose main objective is to fight against the forest fire and distinguish it which includes the earliest possible fire detection methods, the proper classification of fire and also the fast responses that could be from the forest officials. So they are using some modern technologies like Artificial intelligence (AI) and drones which constantly patrol over the fire area. In this they are using two types of drones to detect the fire as soon as possible, one is a fixedwing drone and the other one is a rotary-wing drone. The fixed-wing drone continually patrols the area and observes the forest land below. Since the fixed-wing drone will fly at the medium height $(350 \mathrm{~m}$ to $5500 \mathrm{~m})$, it might report false alarms because of height or lack of an acute sight. If the fixed-wing detects the fire the rotary wing-drone becomes active and is sent to the location detected by the GPS for clear vision and observation. The reason for sending the rotary- wing drone is to minimise the false positive alarms sent to the forest officials.

This proposed system is completely automatic since both drones have impending computers and also their own 


\section{International Journal of Engineering Applied Sciences and Technology, 2020 Vol. 5, Issue 4, ISSN No. 2455-2143, Pages 331-336 \\ Published Online August 2020 in IJEAST (http://www.ijeast.com)}

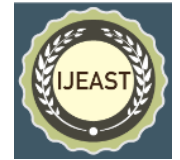

processing efficiencies. They can analyze and detect the fire by using the data recorded by the thermal cameras and this data can be processed without the centralized computing engines. This proposed system has planned to apply Artificial Intelligence by letting the drones make fire predictions using the computer vision techniques to predict the fires they are using neural network concepts which are the network learned by itself with examples without being programmed to predict the fire. The main advantage of the whole process is to predict the fire in less duration time but the disadvantage is it is very costly to implement.

O.R. Vincent, O. Folorunso, et al. in [4] has proposed a paper whose main objective is on Image edge detection which is a process of detecting the edge of an image which is essential in finding the magnitude at each point 'I' on an input image. The edge detection accuracy depends on the algorithm used. The Sobel operator conducts the 2-D spatial gradient measurement on the input images. The Sobel is sensitive to the noise in the images and hence it is able to highlight them as edges.

There are many methods for detecting edges, they are mainly two methods discussed in paper One is gradient and the other is Laplacian. The Sobel operator is an example of the gradient method. The Sobel edge detection algorithm initially accepts the input image, secondly it applies mask, thirdly performs the absolute magnitude of the gradient, and finally gives the detected edges as its output.

Anphy Jose, Deepa Merlin Dixon K, Naiji Joseph \&amp; Silpa George E et al. in [5] has proposed a paper based on the study of the performance of edge detection operators. Image analysis technique includes its processing as postprocessing steps. The changes of intensity in an image is considered as an edge. This paper describes a comparative study among various operators such as Sobel, Prewitt, Roberts \&amp and Canny based on its performance factors.

Miriyala, Trinath \& Karthik, Ragipati \& Mahitha, J \& Reddy, V.et al. in [6] has proposed in his paper that $80 \%$ of fire crisis would have been avoided if the fire was detected immediately. The NodeMCU fire indicator and an observing structure based on IoT technology is the solution to this problem. This paper describes a system containing the Node Mcu which is connected to a temperature sensor, a smoke sensor, and a signal. The temperature sensor is used to detect the heat and the smoke sensor is used to detect any kinds of smoke produced because of the fire. When the fire is detected, the alarm is activated and an alert goes to the respective official. In addition to the alert, the LCD screen also displays messages after the fire is detected. Later the clients or the forest officials can view the details on the Android Page which is very portable and user friendly and helps the clients in analyzing the minute details of the system.

Khalaf, Osamah \& Abdulsaheb, Ghaidaa. et al. [7] In the past few decades many studies and researches have taken place to improve security systems and to increase their level of protection in different fields. One of the major problems that security must deal with is the fire outbreak that can happen in everywhere including houses, schools, factories and many other places, and to avoid that or to minimize the damage caused by fire outbreak an IOT technology is used to control such a kind of risk. IoT is a modern system that consists of sensors and switches connected to a central hub which is called (gateway). In this proposed system using a temperature sensor known as (Flame sensor) with Arduino device to detect fire outbreak and to measure the amount of heat intensity generated by a fire outbreak or in a specific location in our house, offices, and other places.

Ranjith E, Padmabalaji D, Sibisubramanian S, Radhika S et al. in [8] has proposed this paper whose purpose is to design an IoT based system which can be used to detect any forest fire at the earliest before the fire spreads over the large area. This system uses Raspberry pi which is connected to a camera and two sensors. If any kind of calamitous event occurs then the system will immediately get activated and it sends an alert message containing a picture of the region affected by the fire and the location of thedevice.

Vergin Raja Sarobin M, Simrandeep Singh, Abhay Khera, Lakshya Suri, Chhavi Gupta, Ayush Sharma, et al. in [9] told that Fire outbreak has been a common issue in forests and large buildings. In this research drone to detect forest and building fires which uses the techniques of image processing and video processing. Our research can be divided into three main modules. The first module focuses on the cloud service Thing Speak to perform data analysis. The Data collected by the drone, with the help of a flame sensor attached to the Arduino, is transferred to Thing Speak. With the help of an inbuilt camera module, the drone captures real-time images, and image processing is done on that image using google API. The algorithm uses content-based image retrieval and detects whether a fire is caught inside the image or not, along with its intensity. The algorithm also detects the objects present nearby the fire. The third module focuses on video processing. The algorithm detects fire based on the color of the pixels captured. The drone helps in capturing the live update on the area affected. All three modules boost up fire detection accuracy and can thus help in saving lives.

\section{SYSTEM ANALYSIS AND DESIGN}

\section{A.GPS Module}

\section{IR Sensor}

The IR sensor senses the smoke, heat, infrared, or ultraviolet light radiations. The IR sensor works on the concept of analyzing IR signals. When a flame or a fire is burning, it emits IR signals. These IR signals are then received by the IR receiver on the fire sensor module to detect the flame or fire. This sensor has a 


\section{International Journal of Engineering Applied Sciences and Technology, 2020 \\ Vol. 5, Issue 4, ISSN No. 2455-2143, Pages 331-336 \\ Published Online August 2020 in IJEAST (http://www.ijeast.com)}

voltage from 3 volts to 5.5 volts and the detection angle of the sensor is $60^{\circ}$.

\section{Temperature Sensor}

The temperature sensor (LM 35) most commonly detects fire when the temperature goes above 60 degrees Celsius. The temperature is directly calibrated in degrees Celsius (Centigrade). It is suitable for remote applications and operates from 4 to 30 Volts and the most important feature of this component in fire detection is that it is low selfheating.

\section{GPS Module}

The Neo 6M GPS module is a complete GPS (Global Positioning System) receiver with an in built $25 * 25 * 4 \mathrm{~mm}$ antenna made of ceramic. It is used to detect the location of the fire. The latitudinal and longitudinal information is sent to the microcontroller which processes the data and then forwards the coordinates to the person's pre-registered contact number.

\section{SIM800L GSM Module}

The SIM800L GSM module has the most unique feature of Short Message Service (SMS) using the Serial Communication Method. This module helps in automatically sending an SMS when the temperature is increased due to fire. The SMS will contain the URL of the fire detected location. The operating voltage of this module is from 3.5 Volts to 4.4 Volts. This module comes with a helical antenna and Sim socket on the back and any activated $2 \mathrm{G}$ micro sim would work perfectly to send the SMS to the person's pre-registered number.

\section{ESP8266 microcontroller}

ESP 32 Microcontroller is an IoT computer code with a built in ESP-8266 Wi-Fi Module. It is the brain of the entire system which is interfaced with all the other components. This device gathers data from both the sensors and transfers it to the base. This data which is collected is exhibited using the Thingspeak Application. It is also controlled in such a manner that it gives the required notifications which makes the whole monitoring process well organized.

\section{LCD}

The messages in LCD (Liquid Crystal Display) are displayed in 16 characters per line. It is used to display various messages about the tasks happening in the entire detection system. The messages are displayed to help the user know the status of the system to take further action.

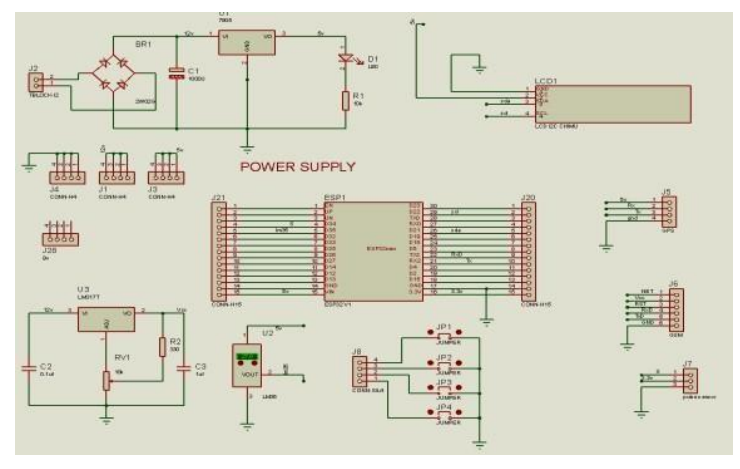

Fig. 1. Schematic Pin Diagram of GPS Module

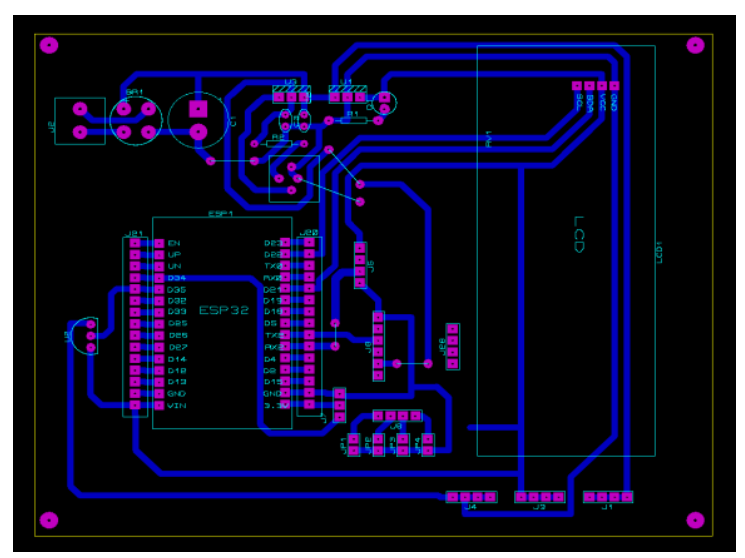

Fig. 2. Layout Design of GPS Module

\section{B. Drone Module}

The drone is an Unmanned Aerial Vehicle which is a flying robot that can be controlled using a remote or the drone can fly uncontrolled through software codes. The Drone is sent to the fire detected location to capture the images. These images are stored in the dataset folder of the Forest officer PC and those will be processed, used to analyze the intensity and direction of the fire using the Sobel Edge Detection Algorithm (Image processing technology)

\section{SYSTEM ARCHITECTURE}

The System Architecture of the entire system is as follows in Fig.3.

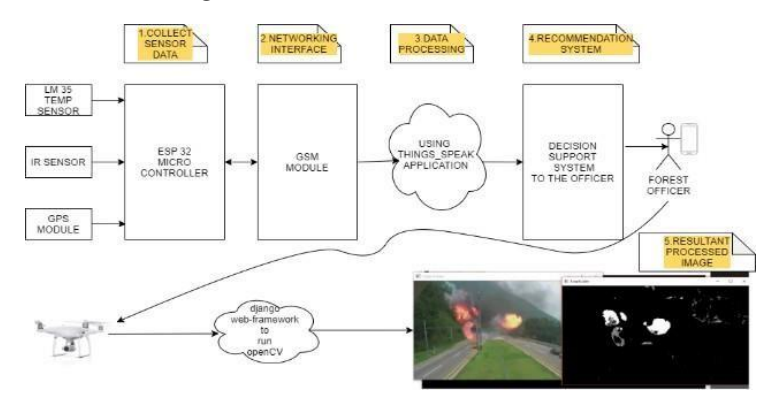

Fig. 3. System Architecture

The above system architecture diagram is an overall view of the forest fire detection system. It is designed using two technologies which are IoT and the Image 


\section{International Journal of Engineering Applied Sciences and Technology, 2020 \\ Vol. 5, Issue 4, ISSN No. 2455-2143, Pages 331-336 \\ Published Online August 2020 in IJEAST (http://www.ijeast.com)}

Processing technology.

The entire proposed system is divided into two parts:

- Module-1: GPS (using IoT Technology)

- Module-2: Drone (using Image

Processing)

The first module contains the hardware documents to perform all the operations. Initially, the temperature and IR sensors are used to detect any fire, hot gases, or flames in the forest. Once any fire is detected this information is sent to the ESP 32 Microcontroller which activates the GPS Module and this will trace the fire detected location.

Once the location is traced, the GPS Module will send this information to the ESP32 Microcontroller and will process it in the form of a URL, and using the Sim800L GSM Module, this URL will be sent to the registered forest officials via an SMS. This information is also stored in a cloud backup called Thingspeak Application which enables the forest officials to register themselves, $\log$ in as users and retrieve this data, analyze the changes in the temperature happening at the fire detected location. This analysis is possible because the LM 35 Temperature sensor is interfaced with the Thingspeak Application.

Once the forest officer opens the link, the screen will open maps which will show the fire detected location. This will help the officer in getting a clear idea about where exactly the fire has caught. After knowing the location, the forest officer will send the drone to this location to capture the images of the fire to analyze its intensity and direction.

The second module which uses the Image Processing technology comes into the picture after the images are captured. This helps in processing the images caught by the drone using the Sobel edge algorithm on the Django web-app which enables the forest officials to register, log in as users, upload the images and process them which helps the forest officials in getting a clear idea about how intense the fire is, the speed in which the fire is spreading and also its direction.

This will help the forest officials to send the right amount of equipment and people to stop the fire from spreading and in extinguishing it

\section{WORKFLOW}

The workflow diagram of the Django application for processing the fire images in Fig 4.

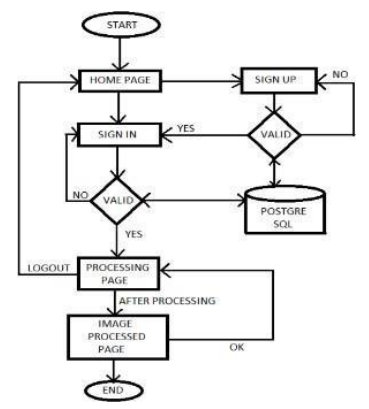

Fig. 4. Flowchart of Django web-app for processing
1. The application starts with a homepage that has three hyperlinks: home, login, sign up.

2. If the officer is new to the forest department he will go to the signup page to create a new account by giving all the details such as username, password, confirm password, etc. and those details are saved in the database called PostgreSQL, In case the officer types different password and confirm password it shows a warning message password not matching and redirects to the same page.

3. Once the forest officer creates the account successfully he can sign in by giving a valid username and password those details are checked from the database. If the given details are valid it will redirect to the processing page In case the officer types invalid username and password it shows a warning message like invalid credentials and redirects to the same page.

4. After an officer logs in, he will be redirected to the processing page where he can browse the image from the image dataset folder for processing and upload it onto the page.

5. Once the image is processed, he can view the processed image in the image processed page.

6. An image processed page after clicking the ok button the page will redirect to the processing page for doing any further image processing on fire images.

7. After his job is done, he can log out by clicking the logout button on the processing page which will redirect to the home page.

The Sobel Edge Detection Algorithm workflow diagram in Fig 5

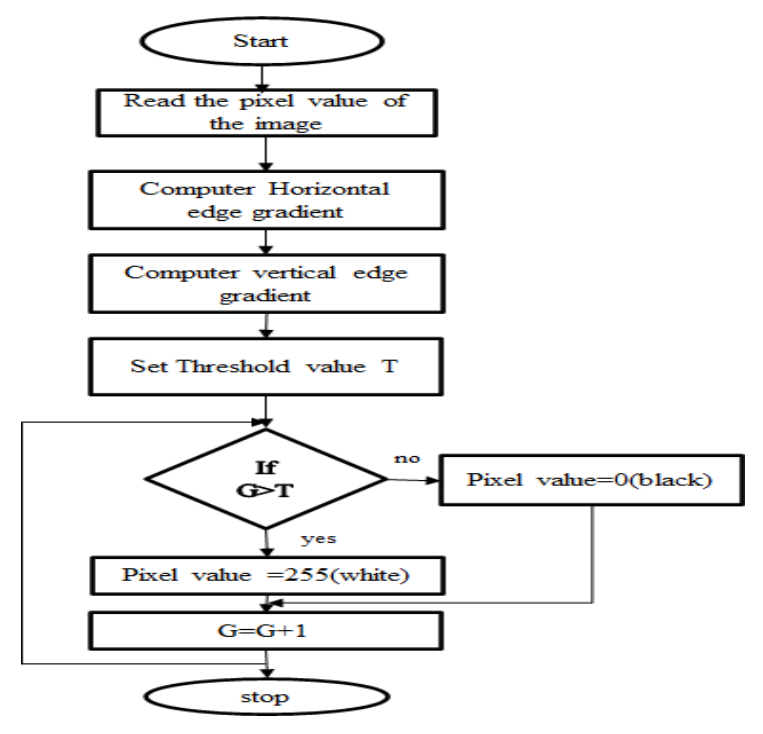

Fig. 5. Flowchart of the Sobel Edge detector algorithm

Sobel Edge Detection algorithm takes the image as an input and converts into the pixels. Applying a mask of Gx, Gy separately on the pixels and forms the absolute 


\section{International Journal of Engineering Applied Sciences and Technology, 2020 Vol. 5, Issue 4, ISSN No. 2455-2143, Pages 331-336 \\ Published Online August 2020 in IJEAST (http://www.ijeast.com)}

gradient. If the absolute gradient is greater than the conditioned threshold value the output pixel becomes white if not it becomes black.

\section{DATA ANALYSIS AND RESULTS}

Data Analysis occurs in the main station. It consists of the following:

\section{A. ThingSpeak}
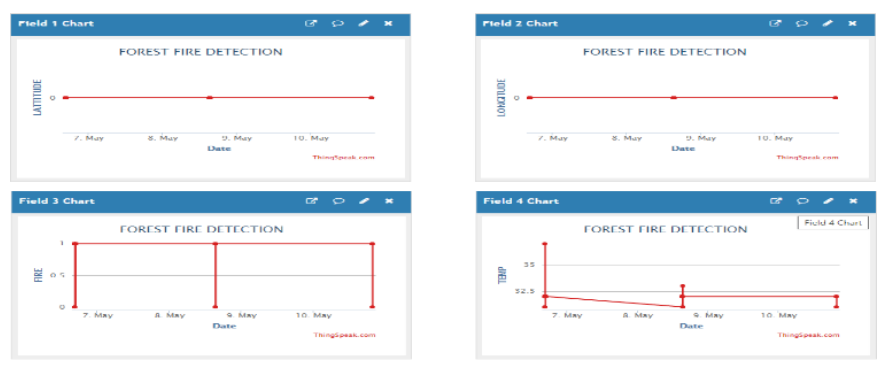

Fig. 6. Thingspeak graphical analysis

Field 1 \& Field 2: With the help of GPS, this channel stores the latitude and longitude value of a device from a particular location.

Field 3: this channel stores the value in terms of 0 and 1. Where 0 represents no detections of fire nearby and 1 represents the detection of fire in the nearby area.

Field 4: this channel stores the temperature of the area, and its variation also helps us analyse the situation.

\section{B. Image Processing}

The real-time images of the fire clicked by the drone camera are acquired and processed using OpenCV in Python on Django Web-app for analysing the intensity and direction of the fire. Based on the visual analysis of the image the required amount of help from the forest officials.

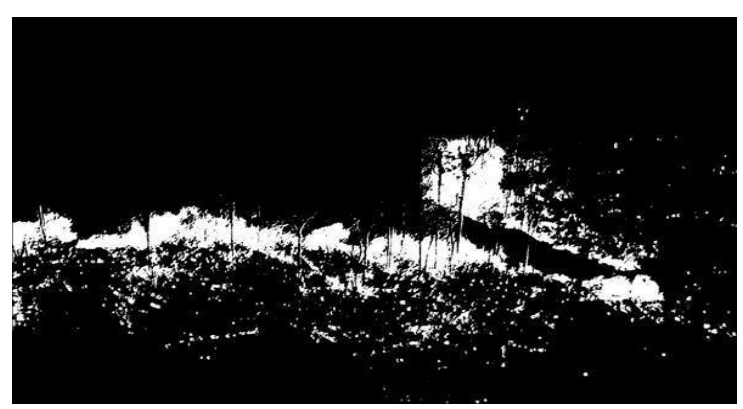

Fig. 7. The processed image on the Django web-app after applying the Sobel edge detection algorithm.

\section{FUTURE SCOPE}

These are some of the unexplored ideas that haven't been covered yet.
1. improvement of the system with more advanced features which will include the enhancement of the range of sensing of the sensors to keep a track on the number of animals present in the forest, and this will help to prevent the animals from being endangered.

2. Using two drones, one for short-range distance and the other for long-range distance for better communication.

3. Drones with on-board computers and processing capabilities which are useful for implementing artificial intelligence like developing neural networks that can identify fire or not in the images and videos.

4. Sending a fire detected location URL to forest officer email whenever the IoT module detects the fire.

\section{CONCLUSION}

Forest fires cause damage to the environment only when they are not detected immediately. When this problem is analyzed and immediate alert will be sent to the forest officials, this will help in avoiding huge environmental losses and cultural heritage damages. Therefore, the key goals in this type of a system are that the whole process is very quick, the detection is reliable to trace the location of the fire. It is easier to distinguish a fire when the starting location of the fire is known, and while it has just begun to spread. The information about how the fire is spreading includes its intensity and direction is very important for the forest officials for managing the fire during its various stages. Depending on this information, the forest officials will be guided to stop the fire before it spreads more by analyzing the required amount of people, equipment, and vehicles to distinguish the fire.

Designed this system which can detect and reduce calamatic events that occur because of fire in the forest. The forest fire detection system's most important feature is its speed that will help the forest officials to get notified about the fires as early as possible to prevent the fire from spreading over a large area.

- The transmission of useful information which helps in earlier fire detection thus reducing the impacts caused to the environment and the ecology.

- Fire detection is very accurate and false SMS alerts are rare if any

- Help is sent to the fire detected location immediately once the location URL is received which reduces the fire to distinguish and not cover a bigger area.

- Disaster information sharing in real-time can minimize the loss of human resources and the habitat of the fauna.

Some of the limitations of the system are images that cannot be sent directly to the Django Web-app for processing and Drone doesn't have the built-in onboard computer in it for developing AI to categorize the elements of the image. 


\section{REFERENCES}

[1] Bosch, Ignacio \& Gomez, Soledad \& Vergara, Luis. (2007). Automatic Forest Surveillance Based on Infrared Sensors. $572-$

577.

10.1109/SENSORCOMM.2007.4394981.

[2] Y. Deshpande, K. Savla, C. Lobo, S. Bhattacharjee, and J. Patel, "Forest Monitoring System Using Sensors, Wireless Communication, and Image Processing," 2018 Fourth International Conference on Computing Communication Control and Automation (ICCUBEA), Pune, India, 2018, pp. 1-6, DOI: 10.1109/ICCUBEA.2018.8697708.

[3]D. Kinaneva, G. Hristov, J. Raychev and P. Zahariev, "Early Forest Fire Detection Using Drones and Artificial Intelligence," 2019 42nd International Convention on Information and Communication Technology, Electronics and Microelectronics (MIPRO), Opatija, Croatia, 2019, pp. 1060-1065, DOI: 10.23919/MIPRO.2019.8756696.

[4] Vincent, Olufunke \& Folorunso, Olusegun. (2009). A Descriptive Algorithm for Sobel Image Edge Detection.

[5] Miriyala, Trinath \& Karthik, Ragipati \& Mahitha, J \& Reddy, V. (2018). IoT based forest fire detection system. International Journal of Engineering \& Technology. 7. 124. 10.14419/ijet. v7i2.7.10277.

[6] Khalaf, Osamah \& Abdulsaheb, Ghaidaa. (2019). IOT fire detection system using a sensor with Arduino_http. AUS. 26. 74-78. 10.4206/aus.2019.n26-7.

[7]RANJITH E, PADMABALAJI D, SIBISUBRAMANIAN S, Ms. RADHIKA S," AN IOT BASED FOREST FIRE DETECTION AND PREVENTION SYSTEM USING RASPBERRY PI 3", International Research Journal of Engineering and Technology (IRJET), Volume: 06 Issue: 03. Mar 2019.

[8] Vergin Raja Sarobin M, Simrandeep Singh, Abhay Khera, Lakshya Suri, Chhavi Gupta, Ayush Sharma," Forest Fire Detection using IoT Enabled Drone", International Journal of Pure and Applied Mathematics Volume 119 No. 12 2018, 2469- 2479 ISSN: 1314-3395. 